\title{
Relación entre destreza musical y lateralización derecha del lenguaje en población zurda
}

\author{
Esteban Villar Rodríguez \\ esteban.villar@uji.es \\ María de los Ángeles Palomar García \\ mpalomar@psb.uji.es \\ Jesús Adrián Ventura \\ jadrian@uji.es \\ Gustau Olcina Sempere \\ golcina@uji.es \\ César Ávila Rivera \\ avila@psb.uji.es
}

\section{Resumen}

Introducción: El lenguaje es una función que se encuentra lateralizada en el hemisferio cerebral izquierdo para la mayoría de la población. No obstante, la lateralidad manual izquierda (ser zurdo) se ha asociado a una mayor incidencia de dominancia atípica (derecha) del lenguaje. Se cree que este efecto estaría provocado por una asimetría interhemisférica en las regiones cerebrales auditivas. Dado que los músicos presentan diferencias cerebrales en algunas de estas regiones en su hemisferio derecho, nos preguntamos si su desarrollo cerebral diferencial podría tener algún impacto sobre su dominancia hemisférica del lenguaje.

Métodos: Evaluamos la dominancia hemisférica del lenguaje en no músicos y músicos diestros y en no músicos y músicos zurdos. Para ello, utilizamos un paradigma de resonancia magnética funcional (fMRI) durante una tarea de generación verbal. Se usó el software BrainVoyager QX para todos los análisis.

Resultados: Los músicos zurdos presentaron una mayor incidencia de dominancia derecha que los no músicos zurdos $(44,1 \%$ frente a $15 \%)$. No se encontraron diferencias en la población diestra.

Conclusiones: La probabilidad de que el procesamiento del lenguaje se encuentre lateralizado en el hemisferio derecho es mayor entre músicos zurdos que en no músicos zurdos. Teniendo en cuenta que la instrucción musical comienza después de establecerse la dominancia del lenguaje, interpretamos estos resultados como un nexo común entre los factores que predisponen a la música y a la dominancia atípica del lenguaje. 
Palabras clave: dominancia del lenguaje, músicos, zurdos, lateralización, resonancia magnética funcional (fMRI).

\section{Abstract}

Introduction: Language is a brain function left-lateralized for the majority of population. However, left-handedness has been associated with a higher probability of presenting atypical (right) dominance for language. It is believed that this effect would be caused by an inter-hemispheric asymmetry in the auditory brain regions. Taking into account that musicians present differences in these brain areas in their right hemisphere, we wondered if their differential brain development could have some impact in their hemispheric dominance of language.

Methods: We assessed hemispheric dominance of language in right-handed nonmusicians and musicians, and in left-handed non-musicians and musicians. In order to do this, we used a functional magnetic resonance imaging (fMRI) paradigm during a verb generation task. BrainVoyager QX software was used for all analysis.

Results: Left-handed musicians presented a higher incidence of right dominance than left-handed non-musicians $(44.1 \%$ vs. $15 \%)$. No differences were found among right-handers.

Conclusions: Language processing has a higher chance of being right-lateralized in left-handed musicians than in left-handed non-musicians. Taking into account that musical training begins once language dominance is already established, we interpret these results as a common nexus between the factors that predispose to music and to atypical language dominance.

Keywords: language dominance, musicians, left-handed, lateralization, functional magnetic resonance imaging ( $\mathrm{fMRI}$ ).

\section{Introducción}

Ya en el siglo XIX, el lenguaje fue la primera función cognitiva de la que se encontró un sustrato neuroanatómico claro. Así, los hallazgos post mortem de Paul Broca y Carl Wernicke en pacientes afásicos permitieron establecer que la función del lenguaje resultaba de la interacción entre el giro temporal superior izquierdo y el giro frontal inferior izquierdo (Tremblay y Dick 2016; véase Price 2012 para un modelo revisado del lenguaje). En consecuencia, el lenguaje se definió (y todavía se define) como una función totalmente lateralizada en el hemisferio cerebral izquierdo. Sin embargo, en la actualidad sabemos que esto no es siempre cierto, ya que existen algunas condiciones que se han asociado a una dominancia cerebral atípica (no izquierda) del lenguaje, como, por ejemplo, la lateralidad manual izquierda, ciertas asimetrías neuroanatómicas (Greve et al. 2013), la epilepsia temprana del lóbulo temporal (Springer et al. 1999) y algunas lesiones cerebrales (Rasmussen y Milner 1977).

La lateralidad manual izquierda (es decir, ser zurdo) ha sido una de las condiciones más estudiadas. Así, procedimientos como el test de Wada o la resonancia magnética funcional (fMRI) han permitido analizar la incidencia de la dominancia atípica del lenguaje (tanto derecha como bilateral) entre la población zurda (Knecht et al. 2000; Pujol, Deus, Losilla y Capdevila 1999; Somers et al. 2015). Los resultados obtenidos son consistentes y sitúan la incidencia de dominancia atípica entre la población zurda 
en aproximadamente un 22-24\%, frente al 4-6\% que suele encontrarse entre la población diestra. Si bien estos resultados demuestran la relación de la lateralidad manual con la dominancia del lenguaje, también apuntan a que la dominancia no puede ser únicamente explicada por este factor, ya que la mayoría de los zurdos siguen presentando una dominancia típica izquierda. Así pues, se propuso la hipótesis de que el verdadero factor subyacente a la dominancia sería la existencia de una asimetría cerebral a favor del hemisferio dominante. Históricamente, se ha puesto especial énfasis en las asimetrías de las distintas divisiones del córtex auditivo (Geschwind y Levitsky 1968), encontrándose recientemente una posible relación entre la asimetría en el giro de Heschl (corteza auditiva primaria) y la dominancia del lenguaje (Tzourio-Mazoyer et al. 2015). De ser cierta esta hipótesis, cabría esperar que aquellas personas con una asimetría en Heschl favorable al hemisferio derecho presentasen una dominancia derecha.

¿Existe alguna condición que se asocie a una asimetría derecha del giro de Heschl? Algunas líneas de investigación apuntan que sí, concretamente en los músicos. Las interacciones auditivo-motoras del hemisferio derecho (análogas a las que, típicamente, posibilitan el lenguaje en el hemisferio izquierdo) han sido propuestas como responsables del procesamiento musical (Zatorre, Chen y Penhune 2007). En consonancia, se ha encontrado que los músicos presentan diferencias tanto estructurales (Halwani, Loui, Rüber y Schlaug 2011) como funcionales (PalomarGarcía, Zatorre, Ventura-Campos, Bueichekú y Ávila 2017) en estas conexiones. Más relevante aún para lo que nos atañe: se ha encontrado también un mayor volumen de sustancia gris en el giro de Heschl derecho de los músicos (Bermudez, Lerch, Evans y Zatorre 2009). Si bien esto último no constituye en sí mismo una evidencia de asimetría interhemisférica favorable a Heschl derecho entre los músicos, sí permite decir que los músicos presentan, como mínimo, una asimetría en Heschl marcadamente menos izquierda que el resto de la población.

Por lo tanto, en base a este mayor volumen de sustancia gris en el giro de Heschl derecho de los músicos, y a la posible relación entre la asimetría en Heschl y la dominancia del lenguaje, planteamos que los músicos deberían mostrar diferencias en la dominancia del lenguaje respecto al resto de la población. En consecuencia, el objetivo de este estudio será analizar la dominancia del lenguaje tanto en músicos como en no músicos, y tanto en población diestra como en población zurda (con el objetivo de controlar el ya conocido efecto de la lateralidad manual). Para ello, analizaremos la actividad cerebral de los participantes mediante resonancia magnética funcional durante una tarea típicamente utilizada para evaluar el lenguaje (Sanjuan et al. 2010). Nuestra hipótesis es que encontraremos una mayor incidencia de dominancia atípica del lenguaje entre los músicos que entre los no músicos, tanto en población diestra como en población zurda. Asimismo, también esperamos seguir encontrando el efecto de la lateralidad manual entre los músicos, reflejándose en una mayor incidencia de dominancia atípica en los músicos zurdos que en los músicos diestros.

\section{Métodos}

\section{Participantes}

Un total de 92 sujetos participaron en el presente estudio. Los participantes se agruparon en función a dos variables: destreza musical (músicos y no músicos) y lateralidad manual (diestros y zurdos). Por tanto, se formaron cuatro grupos: no músicos diestros $(n=20)$, músicos diestros $(n=18)$, no músicos zurdos $(n=20)$ y músicos zurdos $(n=34)$. 
La condición para ser clasificado como músico fue haber recibido formación musical formal (conservatorio o academia) durante al menos nueve años, así como tocar un instrumento musical en la actualidad. Nuestros participantes músicos presentaban una media \pm DT de duración de sus estudios musicales de $10 \pm 1,7$ años. Los no músicos no habían tocado nunca un instrumento ni habían recibido formación musical más allá de la educación escolar básica. La lateralidad manual fue autorreportada por cada participante y seguidamente evaluada mediante el Edinburgh Handedness Inventory (Oldfield 1971). Aquellos participantes categorizados por este inventario como ambidiestros fueron clasificados en función de la mano hábil para escribir (en los dos casos en los que esto fue necesario, coincidió con la lateralidad manual autorreportada por el participante).

No se encontraron diferencias estadísticamente significativas entre los cuatro grupos en sexo $\left(X^{2}=1,494 ; p>0,05\right)$ ni edad $(F=0,771 ; p>0,05)$. Los músicos y no músicos de misma lateralidad manual no difirieron significativamente en la puntuación del Edinburgh Handedness Inventory (diestros: $t=-0,61 ; p>0,05$; zurdos: $t=-1,11$; $p>0,05)$. Puede encontrarse información más detallada sobre los participantes en la tabla 1. Ninguno de los participantes había sufrido un trastorno neurológico o psiquiátrico ni presentaba un historial de traumatismo craneal con pérdida de conciencia. Se obtuvo consentimiento informado por escrito de todos los participantes, siguiendo un protocolo aprobado por la Universitat Jaume I.

Tabla 1

Datos sobre edad, sexo y lateralidad manual de los participantes

\begin{tabular}{|c|c|c|c|c|}
\hline Grupo & $\mathbf{N}$ & $\begin{array}{l}\text { Edad media } \pm \text { DT } \\
(\text { rango) }\end{array}$ & Sexo & $\begin{array}{l}\text { Edinburgh media } \\
\pm \text { DT (rango) }\end{array}$ \\
\hline $\begin{array}{l}\text { Diestros } \\
\text { no músicos }\end{array}$ & 20 & $\begin{array}{l}21,2 \pm 2,4 \\
(18-25)\end{array}$ & $\begin{array}{l}11 \text { hombres, } \\
9 \text { mujeres }\end{array}$ & $\begin{array}{c}16,6 \pm 3,3 \\
(11-22)\end{array}$ \\
\hline $\begin{array}{l}\text { Zurdos } \\
\text { no músicos }\end{array}$ & 20 & $\begin{array}{c}21,2 \pm 2,2 \\
(18-27)\end{array}$ & $\begin{array}{l}12 \text { hombres, } \\
8 \text { mujeres }\end{array}$ & $\begin{array}{c}40,9 \pm 6,7 \\
(26-50)\end{array}$ \\
\hline $\begin{array}{l}\text { Diestros } \\
\text { músicos }\end{array}$ & 18 & $\begin{array}{l}20,4 \pm 1,8 \\
(18-23)\end{array}$ & $\begin{array}{l}11 \text { hombres, } \\
7 \text { mujeres }\end{array}$ & $\begin{array}{l}16,7 \pm 4 \\
(10-22)\end{array}$ \\
\hline $\begin{array}{l}\text { Zurdos } \\
\text { músicos }\end{array}$ & 34 & $\begin{array}{c}20,5 \pm 2 \\
(18-26)\end{array}$ & $\begin{array}{l}24 \text { hombres, } \\
10 \text { mujeres }\end{array}$ & $\begin{array}{c}42,7 \pm 4,9 \\
(30-50)\end{array}$ \\
\hline TOTAL & 92 & $\begin{array}{c}20,7 \pm 2,1 \\
(18-27)\end{array}$ & $\begin{array}{l}58 \text { hombres, } \\
34 \text { mujeres }\end{array}$ & $\begin{array}{l}31,6 \pm 13,5 \\
(10-50)\end{array}$ \\
\hline
\end{tabular}

Tarea de resonancia magnética funcional

Se utilizó una tarea de generación verbal computarizada y adaptada para escáneres de resonancia magnética. Esta tarea se encuentra descrita con mayor detalle en Sanjuán et al. (2010). En resumen, la tarea consta de dos condiciones: control y activación. Durante los bloques de control, se presentan consecutivamente y por escrito parejas de letras al participante y este debe leerlas en voz alta. Durante los bloques de activación, se presentan consecutivamente y por escrito sustantivos concretos al participante y este debe responder en voz alta el primer verbo que le venga a la mente para cada sustantivo. 


\section{Adquisición y procesado de datos de resonancia magnética}

Las imágenes fueron adquiridas en un escáner Philips Achieva de 3-T. Se adquirió una imagen de resonancia magnética estructural $3 \mathrm{D}$ de cada participante utilizando una secuencia MPRAGE potenciada en T1 (TR/TE = 8,4/3,8 ms; matriz = $320 \times 320 \times 250$; tamaño del vóxel $=0,75 \times 0,75 \times 0,8 \mathrm{~mm})$. Para la imagen de resonancia magnética funcional, se adquirieron 150 volúmenes utilizando una secuencia EPI potenciada en T2* $($ TR/TE $=2500 / 80 \mathrm{~ms} ;$ matriz $=80 \times 80$; tamaño del vóxel $=3 \times 3 \times 4 \mathrm{~mm})$. Se adquirieron 31 cortes axiales intercalados y alineados a la intersección entre las comisuras anterior y posterior (AC-PC), cubriendo todo el cerebro.

Las imágenes adquiridas fueron procesadas y analizadas mediante el software BrainVoyager QX versión 2.8 (Goebel, Esposito y Formisano 2006). El preprocesado aplicado fue el estándar e incluyó los siguientes pasos: corrección lineal del tiempo de escaneado de los cortes, corrección 3D trilineal del movimiento, suavizado espacial FWHM de $6 \mathrm{~mm}$ y filtrado temporal de paso alto. Las imágenes no fueron normalizadas a ninguna plantilla cerebral. Posteriormente, se corregistró tanto automática como manualmente la imagen estructural T1 de cada participante a sus imágenes funcionales. Una vez definido el modelo lineal general (GLM) correspondiente a la tarea de generación verbal, las imágenes funcionales de cada participante fueron visualizadas bajo los siguientes parámetros: señal BOLD positiva (condición de activación), $\mathrm{k}=40$ y umbrales $\mathrm{FDR}=0,001 ; 0,005$ y 0,01 (es decir, se visualizaron tres sets de imágenes funcionales para cada participante bajo tres umbrales correctores distintos). En el caso de que un participante no mostrase actividad cerebral relevante para una tarea de lenguaje en ninguno de los umbrales anteriormente descritos, se le aplicó un umbral de FDR adicional de 0,05 (solo 4 participantes necesitaron de este umbral extra).

Análisis de la dominancia del lenguaje

Las imágenes funcionales obtenidas fueron utilizadas para evaluar la dominancia del lenguaje de cada participante. Para ello, se valoró qué hemisferio mostraba actividad cerebral significativa durante la tarea de lenguaje en las áreas de Brodmann 44 y 45 (área de Broca), regiones críticas para la producción y articulación del lenguaje y muy representativas de esta función durante la tarea funcional utilizada (Sanjuán et al. 2010). Por lo tanto, se visualizaron los tres sets de imágenes funcionales pertenecientes a cada participante (un set por cada umbral de FDR) y, para cada set, se emitió un juicio independiente de entre dos posibles: dominancia izquierda (actividad cerebral en Broca izquierdo) o dominancia derecha (actividad cerebral en Broca derecho). En aquellos casos en los que se podía observar actividad cerebral significativa en las áreas de Broca de ambos hemisferios, se optaba por aquel hemisferio que mostrase el clúster de activación con mayor significación estadística. Una vez emitido el juicio para los tres umbrales de FDR de un participante, se categorizaba la dominancia del lenguaje para ese participante en función del juicio más frecuente. A modo ilustrativo, en la figura 1 pueden verse algunos ejemplos de imágenes funcionales y su correspondiente valoración.

Todo el procedimiento desde el preprocesado de las imágenes hasta el análisis de la dominancia fue llevado a cabo bajo condición de ciego: los investigadores no conocían la lateralidad manual ni la destreza musical de cada participante. Además, el procedimiento de análisis de dominancia anteriormente descrito fue llevado a cabo de manera independiente por dos investigadores diferentes, obteniendo un elevado nivel de acuerdo interjueces ( $k$ de Cohen $=0,82 ; p<0,001$ ). 

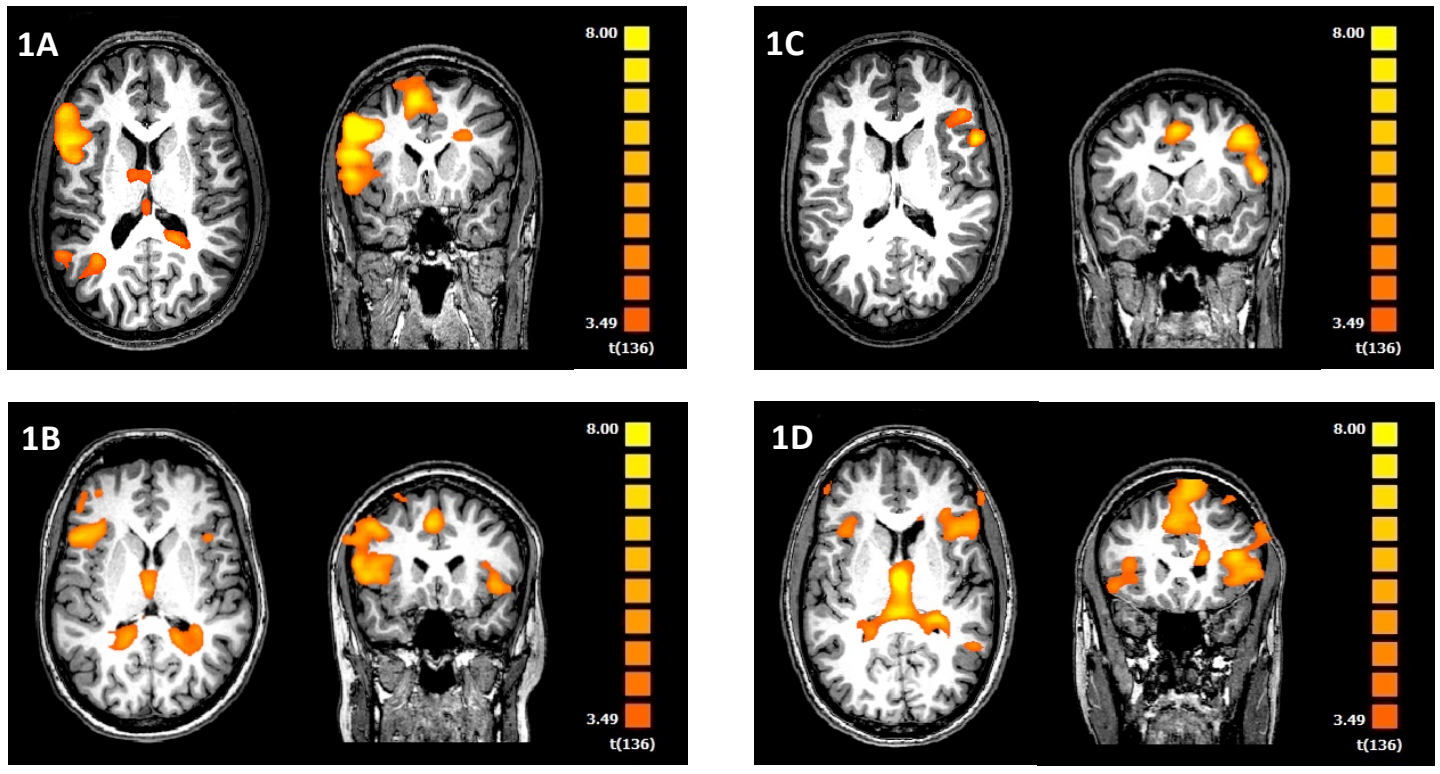

Figura 1. Ejemplos de imágenes valoradas durante el análisis de la dominancia del lenguaje. Las imágenes $1 \mathrm{~A}$ y $1 \mathrm{~B}$ corresponden a una dominancia izquierda, mientras que las imágenes 1C y 1D corresponden a una dominancia derecha.

\section{Análisis estadístico}

En primer lugar, se llevó a cabo una prueba chi cuadrado en una tabla de contingencia $2 \times 2 \times 2$ con el objetivo de poner a prueba la independencia entre las variables de lateralidad manual (diestro/zurdo), destreza musical (no músico / músico) y dominancia del lenguaje (izquierda/derecha).

A continuación, analizamos la distribución de la dominancia del lenguaje en función de la lateralidad manual y la destreza musical. Para ello, aplicamos pruebas chi cuadrado en las tablas de contingencia $2 \times 2$ formadas por: 1) diestros no músicos y diestros músicos; 2) zurdos no músicos y zurdos músicos; y 3) músicos diestros y músicos zurdos. En la prueba número uno, debido a que más del $25 \%$ de las celdas presentaban una frecuencia esperada menor a 5, se sustituyó la prueba chi cuadrado por una prueba exacta de Fisher para un mayor rigor estadístico.

Finalmente, se aplicaron una serie de pruebas post-hoc en búsqueda de posibles factores relacionados con la dominancia del lenguaje. Así pues, se llevaron a cabo pruebas $t$ para muestras independientes entre los músicos zurdos con dominancia izquierda y los músicos zurdos con dominancia derecha. Las variables testeadas fueron las siguientes: edad, puntuación del Edinburgh Handedness Inventory, edad de inicio del entrenamiento musical y total de horas de práctica musical (tanto durante toda la vida como durante la franja de edad de 0 a 7 años). También se comprobaron posibles diferencias por sexo mediante una prueba exacta de Fisher. Solo se exploraron los músicos zurdos porque fueron el único grupo que presentó una distribución de la dominancia lo suficientemente heterogénea como para conformar dos subgrupos de dominancia con un similar tamaño muestral. 


\section{Resultados}

Los resultados del análisis de la dominancia del lenguaje pueden encontrarse en la tabla 2. En resumen: 1) todos los no músicos diestros presentaron dominancia izquierda; 2) la mayoría de músicos diestros presentaron dominancia izquierda, a excepción de 2 participantes; 3) similarmente, los no músicos zurdos también presentaron en su mayoría dominancia izquierda, a excepción de 3 participantes; y 4) los no músicos zurdos mostraron la distribución de dominancia del lenguaje más heterogénea, con 19 participantes con dominancia izquierda y 15 participantes con dominancia derecha.

\section{Tabla 2}

Distribución de la dominancia del lenguaje entre los cuatro grupos

\begin{tabular}{llcc}
\hline $\begin{array}{l}\text { Lateralidad } \\
\text { manual }\end{array}$ & $\begin{array}{l}\text { Destreza } \\
\text { musical }\end{array}$ & $\begin{array}{c}\text { Dominancia } \\
\text { izquierda }\end{array}$ & $\begin{array}{c}\text { Dominancia } \\
\text { derecha }\end{array}$ \\
\cline { 3 - 4 } & No músicos & $\begin{array}{c}\mathrm{N}(\% \text { del } \\
\text { grupo })\end{array}$ & $\mathrm{N}(\%$ del grupo $)$ \\
\hline \multirow{2}{*}{ Diestros } & $\begin{array}{c}20 \\
(100 \%)\end{array}$ & - \\
& Músicos & 16 & 2 \\
& No músicos & 17 & $(11,1 \%)$ \\
Zurdos & Músicos & $(85 \%)$ & 3 \\
& & $19 \%)$ & 15 \\
& & $(55,9 \%)$ & $(44,1 \%)$ \\
\hline
\end{tabular}

La independencia entre lateralidad manual, destreza musical y dominancia del lenguaje fue rechazada $\left(x^{2}=21,52 ; p<0,001\right)$. No se pudo rechazar la distribución homogénea de la dominancia entre diestros no músicos y diestros músicos ( $p$ exacta de Fisher $=0,218$ ). Sí se rechazó la distribución homogénea de la dominancia entre zurdos no músicos y zurdos músicos $\left(x^{2}=4,47 ; p<0,05\right)$, habiendo una mayor incidencia de dominancias derechas entre los zurdos no músicos con una odds ratio de 4,47 (95\% IC =1,1-18,17). También se rechazó la distribución homogénea de la dominancia entre músicos diestros y músicos zurdos $\left(x^{2}=5,83 ; p<0,05\right)$, habiendo una mayor incidencia de dominancias derechas entre los músicos zurdos con una odds ratio de $6,32(95 \% / C=1,25-31,86)$.

Ninguno de los análisis post-hoc realizados arrojó diferencias significativas entre músicos zurdos con dominancia izquierda y músicos zurdos con dominancia derecha.

\section{Discusión y conclusiones}

El objetivo de este estudio era comprobar si existe alguna relación entre ser músico y presentar una dominancia hemisférica del lenguaje atípica (derecha). Los resultados obtenidos apuntan a que existe una mayor incidencia de dominancia derecha del lenguaje entre músicos que entre no músicos en la población zurda (pero no en la 
población diestra) y entre músicos zurdos que entre músicos diestros. Ninguna de las variables exploradas post-hoc entre los músicos zurdos han podido relacionarse con la dominancia del lenguaje presentada.

La mayor proporción de lateralizaciones derechas del lenguaje entre zurdos músicos que entre zurdos no músicos sugiere que, hasta cierto punto, existe una relación entre destreza musical y dominancia del lenguaje. Esta relación, además, no sería pequeña: nuestros resultados muestran que los zurdos tienen hasta 4 veces más probabilidades de presentar una dominancia derecha si, además de zurdos, son músicos. Creemos que este efecto se sustentaría en las diferencias cerebrales presentes en la red auditivo-motora derecha de los músicos. En primer lugar, hay que tener en cuenta que la dominancia del lenguaje rara vez es absoluta y que, en la mayoría de ocasiones, se puede encontrar cierta actividad del hemisferio no dominante (normalmente el derecho) durante tareas de procesamiento lingüístico (Knecht et al. 2000; Pujol, Deus, Losilla y Capdevila 1999; Somers et al. 2015). Esto nos dice que la red auditivo-motora derecha es capaz de involucrarse en los procesamientos lingüísticos ( $y$, de hecho, lo hace con frecuencia). Por lo tanto, planteamos que las diferencias tanto estructurales (Bermudez, Lerch, Evans y Zatorre 2009; Halwani, Loui, Rüber y Schlaug 2011) como funcionales (Palomar-García, Zatorre, Ventura-Campos, Bueichekú y Ávila 2017) presentes en la red auditivo-motora derecha de los músicos podrían relacionarse con una mayor saliencia y participación de esta red durante el procesamiento del lenguaje, pudiendo llegar incluso a sobrepasar a su homóloga izquierda. Concretamente, proponemos que una posible relación entre la dominancia derecha del lenguaje en músicos y su mayor volumen del giro de Heschl derecho (Bermudez, Lerch, Evans y Zatorre 2009) encajaría dentro de la hipótesis de la asimetría interhemisférica de las áreas auditivas como factor explicativo de la dominancia (Geschwind y Levitsky 1968; Tzourio-Mazoyer et al. 2015). También es interesante destacar que Palomar-García, Zatorre, Ventura-Campos, Bueichekú y Ávila (2017) ya señalaron que la red auditivomotora derecha de los músicos muestra una mayor conectividad funcional en estado de reposo, lo que encajaría con encontrar una mayor representación funcional de este circuito también durante las tareas que le son propias, como es el procesamiento auditivo-motor llevado a cabo en el lenguaje.

En cualquier caso, el hecho de que no hayamos encontrado resultados similares en la población diestra nos dice que la relación entre destreza musical y dominancia del lenguaje no es tan simple. La lateralidad manual jugaría un papel crucial en esta interacción, pues únicamente permitiría que este efecto tuviese lugar en la población zurda. Sin embargo, debido al reducido número de participantes diestros presentes en nuestra muestra (20 no músicos y 18 músicos), en conjunción con la bajísima incidencia de dominancia derecha (4-6 \%) documentada para la población diestra en investigaciones previas (Knecht et al. 2000; Pujol, Deus, Losilla y Capdevila 1999; Somers et al. 2015), recomendamos replicar el estudio utilizando una muestra de mayor tamaño. Esto permitiría confirmar que la relación entre destreza musical y dominancia del lenguaje no pueda haber quedado camuflada en la población diestra bajo su reducidísima probabilidad de presentar una dominancia derecha.

Finalmente, las implicaciones de estos resultados dependen en gran medida de cómo interpretamos la causalidad de la relación lenguaje-música: ¿estamos ante un efecto del entrenamiento musical sobre la dominancia cerebral del lenguaje $o$ ante un efecto de la dominancia cerebral del lenguaje sobre la posibilidad de acabar deviniendo músico? Por un lado, el posible efecto de la música sobre el lenguaje estaría en consonancia con investigaciones que han mostrado unas mejores habilidades lingüísticas en niños que han recibido entrenamiento musical (Douglas y Willatts 1994; Tierney y Kraus 2013). Bajo esta interpretación, cabría esperar que estas mejoras lingüísticas se asociasen a una mayor participación de la red auditivo-motora derecha, 
lo que apoyaría esta idea de que existe una transferencia de las habilidades musicales hacia las habilidades lingüísticas. Por otro lado, un efecto del lenguaje sobre la aptitud musical podría estar relacionado con el mayor rol del córtex auditivo derecho en el procesamiento de estímulos tonales o melódicos (Sininger y Bhatara 2012). Así pues, planteamos que una dominancia innatamente derecha del lenguaje podría asociarse a un procesamiento más eficaz de los estímulos musicales y, por tanto, una mayor capacidad musical. De confirmarse este nexo entre dominancia derecha del lenguaje y mayor aptitud musical, se abriría la puerta al uso del análisis de la dominancia como un posible indicador del talento musical en los procesos de selección de las academias musicales. Dadas ambas interpretaciones, si bien el diseño transversal del presente estudio no responde a cuál es la correcta, sí podemos valorar la coherencia de estas interpretaciones con nuestros resultados. Así pues, es pertinente destacar que: 1) los participantes músicos más precoces de nuestro estudio iniciaron su formación musical a los 6 años, una edad a la que supuestamente la dominancia del lenguaje ya está establecida y se vuelve poco modulable salvo lesión cerebral (Szaflarski et al. 2012); y 2) no encontramos diferencias en la dominancia en función de la edad de inicio ni la intensidad del entrenamiento musical. Esto nos sugiere que la formación musical no está modulando la dominancia, sino que nos encontramos más bien ante un nexo de unión entre los factores que predisponen tanto al desarrollo musical como al desarrollo de una dominancia hemisférica derecha del lenguaje. En cualquier caso, estemos ante una dirección causal u otra, estos resultados sugieren la utilidad de recoger en los historiales clínicos de la población si son músicos o no. Y es que, que la lateralidad manual sea hoy en día un dato clínico relevante, se debe a su relación con una organización atípica de algunas funciones cerebrales (como ocurre con el lenguaje). Por tanto, en base a los resultados obtenidos, saber si alguien es músico también podría dar información clínicamente relevante al personal sanitario.

En conclusión, nuestro estudio muestra que la destreza musical se asocia a una mayor incidencia de dominancia atípica (derecha) del lenguaje en población zurda. Proponemos que las diferencias cerebrales propias de los músicos en su red auditivomotora derecha serían responsables de este efecto, predisponiendo tanto al desarrollo musical como a una dominancia hemisférica derecha del lenguaje.

\section{Referencias bibliográficas}

Bermudez, Patrick, Jason P. Lerch, Alan Charles Evans y Robert Zatorre. 2009. «Neuroanatomical correlates of musicianship as revealed by cortical thickness and voxel-based morphometry". Cerebral Cortex 19(7): 1583-1596.

Douglas, Sheila y Peter Willatts. 1994. "The relationship between musical ability and literacy skills ». Journal of Research in Reading 17(2): 99-107.

Geschwind, Norman y Walter Levitsky. 1968. «Human brain: left-right asymmetries in temporal speech region». Science 161: 186-187.

Goebel, Rainer, Fabrizio Esposito y Elia Formisano. 2006. «Analysis of FIAC data with BrainVoyager QX: From single subject to cortically aligned group GLM analysis and self-organizing group ICA». Human Brain Mapping 27(5): 392-401.

Greve, Douglas N., Lise Van der Haegen, Qing Cai, Steven M. Stufflebeam, Mert R. Sabuncu, Bruce Fischl y Marc Brysbaert. 2013. «A surface-based analysis of language lateralization and cortical asymmetry». Journal of Cognitive Neuroscience 25(9): 1477-1492.

Halwani, Gus, Psyche Loui, Theodor Rüber y Gottfried Schlaug. 2011. «Effects of practice and experience on the arcuate fasciculus: Comparing singers, instrumentalists, and nonmusicians». Frontiers in Psychology 2(JUL): 1-9.

Knecht, Stephan, Bianca Drager, Michael Deppe, L. Bobe, Hubertus Lohmann, Agnes Flöel, Erich Bernd Ringelstein y Henning Henningsen. 2000. «Handedness and 
hemispheric language dominance in healthy humans». Brain $123 \mathrm{Pt}$ 12(12): 2512-2518.

Oldfield, Richard Charles. 1971. «The assessment and analysis of handedness: The Edinburgh inventory». Neuropsychologia 9(1): 97-113.

Palomar-García, María Ángeles, Robert J. Zatorre, Noelia Ventura-Campos, Elisenda Bueichekú y César Ávila. 2017. "Modulation of Functional Connectivity in Auditory-Motor Networks in Musicians Compared with Nonmusicians». Cerebral Cortex 27(5): 2768-2778.

Price, Cathy J. 2012. "A review and synthesis of the first 20 years of PET and fMRI studies of heard speech, spoken language and reading». Neurolmage 62(2): 816-847.

Pujol, Jesús, Joan Deus, Josep Maria Losilla y Antoni Capdevila. 1999. "Cerebral lateralization of language in normal left-handed people studied by functional MRI». Neurology 52(5): 1038-1043.

Rasmussen, Theodore y Brenda Milner. 1977. "The Role of Early Left-Brain Injury in Determining Lateralization of Cerebral Speech Functions». Annals of the New York Academy of Sciences 299(1): 355-369.

Sanjuan, Ana, Juan Carlos Bustamante, Cristina Forn, Noelia Ventura-Campos, Alfonso Barrós-Loscertales, Juan Carlos Martinez, Vicente Villanueva y Cesar Avila. 2010. "Comparison of two fMRI tasks for the evaluation of the expressive language function». Neuroradiology 52(5): 407-415.

Sininger, Yvonne S. y Anjali Bhatara. 2012. "Laterality of basic auditory perception». Laterality 17(2): 129-149.

Somers, Metten, Maartje F. Aukes, Roel A. Ophoff, Marco P. Boks, Willemien Fleer, Kees C. L. de Visser, René S. Kahn e Iris E. C. Sommer. 2015. "On the relationship between degree of hand-preference and degree of language lateralization». Brain and Language 144: 10-15.

Springer, Jane A., Jeffrey R. Binder, Thomas Alan Hammeke, Sara J. Swanson, Julie Anne Frost Bellgowan, Patrick Bellgowan, Cameron Craig Brewer, Holly M. Perry, George L. Morris y Wade M. Mueller. 1999. «Language dominance in neurologically normal and epilepsy subjects. A functional MRI study». Brain 122(11): 2033-2045.

Szaflarski, Jerzy P., Akila Rajagopal, Mekibib Altaye, Anna W. Byars, Lisa M. Jacola, Vicent Schmithorst, Mark B. Schapiro, Elena Plante y Scott K. Holland. 2012. «Left-handedness and language lateralization in children». Brain Research 1433: 85-97.

Tierney, Adam y Nina Kraus. 2013. «Music training for the development of reading skills». En Progress in Brain Research, ed. M. Merzenich, M. Nahum y M. Van Vleet. Elsevier B.V.

Tremblay, Pascale y Anthony Steven Dick. 2016. «Broca and Wernicke are dead, or moving past the classic model of language neurobiology». Brain and Language 162: $60-71$.

Tzourio-Mazoyer, Nathalie, Damien Marie, Laure Zago, Gaël Jobard, Guy Perchey, Gaëlle Leroux, Emmanuel Mellet, Joliot Marc, Fabrice Crivello, Laurent Petit y Bernard Mazoyer. 2015. «Heschl's gyrification pattern is related to speech listening hemispheric lateralization: FMRI investigation in 281 healthy volunteers ». Brain Structure \& Function 220: 1585-1599.

Zatorre, Robert J., Joyce L. Chen y Virginia B. Penhune. 2007. «When the brain plays music: auditory-motor interactions in music perception and production». Nature Reviews. Neuroscience 8(7): 547-558. 